\title{
INTEGRAL observations of the field of the BL Lacertae object S5 $0716+714^{\star}$
}

E. Pian ${ }^{1,2}$, L. Foschini ${ }^{2}$, V. Beckmann ${ }^{3,4}$, A. Sillanpää ${ }^{5}$, S. Soldi' ${ }^{6}$, G. Tagliaferri ${ }^{7}$, L. Takalo ${ }^{5}$, P. Barr ${ }^{8}$, G. Ghisellini ${ }^{7}$, G. Malaguti ${ }^{2}$, L. Maraschi ${ }^{9}$, G. G. C. Palumbo ${ }^{10}$, A. Treves ${ }^{11}$, T. J.-L. Courvoisier ${ }^{6,12}$, G. Di Cocco ${ }^{2}$, N. Gehrels ${ }^{3}$, P. Giommi ${ }^{13}$, R. Hudec ${ }^{14}$, E. Lindfors ${ }^{5}$, A. Marcowith ${ }^{15}$, K. Nilsson ${ }^{5}$, M. Pasanen ${ }^{5}$, T. Pursimo ${ }^{16}$, C. M. Raiteri ${ }^{17}$,

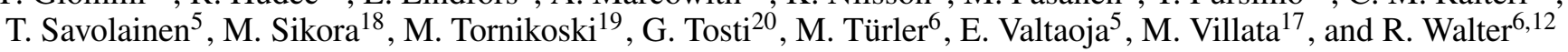

1 INAF - Osservatorio Astronomico di Trieste, via GB Tiepolo 11, 34131 Trieste, Italy e-mail: pian@ts.astro.it

2 IASF-CNR, Sezione di Bologna, via Gobetti 101, 40129 Bologna, Italy

3 NASA Goddard Space Flight Center, Code 661, Greenbelt, MD 20771, USA

4 Joint Center for Astrophysics, Department of Physics, University of Maryland, Baltimore County, MD 21250, USA

5 Tuorla Observatory, University of Turku, 21500 Piikkiö, Finland

6 INTEGRAL Science Data Center, Chemin d'Écogia 16, 1290 Versoix, Switzerland

7 INAF - Osservatorio Astronomico di Brera, via E Bianchi, 46, 23807 Merate (LC), Italy

8 ESA-ESTEC, RSSD, Keplerlaan 1, Postbus 299, 2200 AG Noordwijk, The Netherlands

9 INAF - Osservatorio Astronomico di Brera, via Brera 28, 20121 Milano, Italy

${ }^{10}$ Department of Astronomy, University of Bologna, via Ranzani 1, 40127 Bologna, Italy

11 Dipartimento di Fisica e Matematica, University of Insubria, via Valleggio 11, 22100 Como, Italy

12 Observatoire de Genève, 51 Ch. des Maillettes, 1290 Sauverny, Switzerland

13 ASI Science Data Center, via Galileo Galilei, 00044 Frascati, Italy

14 Astronomical Institute, 25165 Ondrejov, Czech Republic

15 Centre d'Étude Spatiale de Rayonnements, 31028 Toulouse, France

16 Nordic Optical Telescope, Apartado 474, 38700 Santa Cruz de La Palma, Spain

17 INAF - Osservatorio Astronomico di Torino, via Osservatorio 20, 10025 Pino Torinese (TO), Italy

18 Nikolaus Copernicus Astronomical Center, Bartycka 18, 00-716 Warsaw, Poland

19 Metsähovi Radio Observatory, Metsähovintie 114, 02540 Kylmälä, Finland

20 Department of Physics, University of Perugia, via A Pascoli, 06123 Perugia, Italy

Received 20 August 2004 / Accepted 26 August 2004

\begin{abstract}
We have performed observations of the blazar S5 0716+714 with INTEGRAL on 2-6 April 2004. In the first months of 2004, the source had increased steadily in optical brightness and had undergone two outbursts. During the latter outburst, that occurred in March, it reached the extreme level of $R=12.1 \mathrm{mag}$, which triggered our INTEGRAL program. The target has been detected with IBIS/ISGRI up to $60 \mathrm{keV}$, with a flux of $\sim 3 \times 10^{-11} \mathrm{erg} \mathrm{s}^{-1} \mathrm{~cm}^{-2}$ in the 30-60 keV interval, a factor of $\sim 2$ higher than observed by the BeppoSAX PDS in October 2000. In the field of S5 0716+714 we have also detected the Flat Spectrum Radio Quasar S5 0836+710 and the two Seyfert galaxies Mkn 3 and Mkn 6. Their IBIS/ISGRI spectra are rather flat, albeit consistent with those measured by BeppoSAX. In the spectrum of Mkn 3 we find some evidence of a break between 60 and $\sim 100 \mathrm{keV}$, reminiscent of the high energy cut-offs observed in other Seyfert galaxies. This is the first report of INTEGRAL spectra of weak Active Galactic Nuclei.
\end{abstract}

Key words. galaxies: active - gamma-rays: observations

\section{Introduction}

* Based on observations obtained with INTEGRAL, an ESA project with instruments and science data center funded by ESA member states (especially the PI countries: Denmark, France, Germany, Italy, Switzerland, Spain, Czech Republic and Poland), and with the participation of Russia and the USA.

The high energy emission of Active Galactic Nuclei (AGN) carries the most direct and constraining information on the radiation mechanisms and the nature of the central engine. Blazar type AGNs, traditionally subdivided in Flat Spectrum Radio Quasars (FSRQ) and BL Lacertae Objects 
(Urry \& Padovani 1995), are very powerful and variable multiwavelength emitters.

At gamma-ray wavelengths, their spectral output has often a maximum and their variability exhibits the largest amplitudes (Ulrich et al. 1997), making them suitable targets for the INTEGRAL mission, particularly during active states. The radio-quiet and less luminous Seyferts exhibit hard X-ray spectra often extending to the soft gamma-ray domain. At these energies spectral cut-offs have been detected in many of these objects by soft gamma-ray experiments like the CGRO OSSE and the BeppoSAX PDS. Studying these spectral features is relevant to the identification of the emission mechanism responsible for the production of the spectrum at those energies (e.g., Svensson 1996; Haardt et al. 1997; Petrucci et al. 2000).

The BL Lac object S5 $0716+714$ has been monitored at radio and optical wavelengths by more than 40 telescopes in the northern hemisphere during a Whole Earth Blazar Telescope (Villata et al. 2004) campaign lasting from September 2003 to June 2004. The source had been already observed by INTEGRAL in October 2003 during an optically active state (Wagner et al. 2004, in prep.). In January and March 2004, S5 0716+714 was in outburst and achieved its optical historical maximum. In late March 2004 it brightened by 2 magnitudes with respect to its November 2003 level, and by 1 magnitude in $\sim 2$ weeks, reaching a magnitude of 12.1 in the optical $R$-band (Sillanpää et al. 2004, in prep.).

The large optical variation observed in March 2004 matched the trigger criteria for our INTEGRAL Target-ofOpportunity program for blazars in outburst (Proposal ID: 220049), thus we activated the campaign. Observations with $R X T E$ and XMM-Newton as well as with ground-based optical and radio telescopes have been carried out simultaneously with INTEGRAL. The results of this multiwavelength monitoring will be reported in forthcoming papers. We report here on the INTEGRAL observations of the field of S5 0716+714, in which we have also detected another blazar and two Seyfert galaxies.

\section{Observations, data analysis and results}

INTEGRAL (Winkler et al. 2003) observed S5 0716+714 (Galactic coordinates: $l=144^{\circ}, b=+28^{\circ}$ ) starting from 2004 April $2^{\text {nd }}, 20: 49: 25$, and ending on 2004 April $7^{\text {th }}, 00: 14: 08$ UT. In order to optimize the performance of the SPI spectrometer (Vedrenne et al. 2003) we adopted an observing scheme consisting of one pointing of about $2 \mathrm{ks}$, followed by a slew of $120 \mathrm{~s}$, then by another pointing, and so on, so as to build a rectangular pattern (dither pattern $5 \times 5$ ).

The total duration of the observation was of $280 \mathrm{ks}$, but the effective exposures of the IBIS/ISGRI (Ubertini et al. 2003; Lebrun et al. 2003), IBIS/PICsIT (Ubertini et al. 2003; Di Cocco et al. 2003), SPI (Vedrenne et al. 2003) and JEM-X (Lund et al. 2003) detectors were of 256, 237, 218, and 189 $\mathrm{ks}$, respectively (only JEM-X1 was used, while JEM-X2 was switched off). This reduction is due to telemetry gaps and dead time corrections (generally affecting all INTEGRAL observations), to the occurrence of a failure of the VETO module n. 15, that caused IBIS to be idle for $9 \mathrm{ks}$, and to the removal of 3 pointings with $13 \mathrm{ks}$ from the SPI data set because of problems in fitting them.

The screening, reduction, and analysis of the INTEGRAL data have been performed using the INTEGRAL Offline Scientific Analysis (OSA) V. 4.0, publicly available through the INTEGRAL Science Data Center ${ }^{1}$ (ISDC, Courvoisier et al. 2003a). The algorithms implemented in the software are described in Goldwurm et al. (2003) for IBIS, Westergaard et al. (2003) for JEM-X, and Diehl et al. (2003) for SPI, and we refer the reader to these papers for more details on the data processing and deconvolution of coded-mask telescopes on board INTEGRAL.

Only the observations with the IBIS/ISGRI instrument yielded significant source detections, as reported in Sects. 2.1 and 2.2. The IBIS/PICsIT, SPI and JEM-X data were also accumulated into final images. However, no sources are detected in those data.

The upper limit for IBIS/PICsIT is $7.3 \times 10^{-10} \mathrm{erg} \mathrm{s}^{-1} \mathrm{~cm}^{-2}$ in the $252-336 \mathrm{keV}$ range $(292 \mathrm{mCrab})$. In the most sensitive SPI energy range, 20-40 keV, the spectrometer achieved a marginally significant detection for Mkn 3: $8.3 \pm 3.5 \times 10^{-4} \mathrm{ph} \mathrm{s}^{-1} \mathrm{~cm}^{-2}$ (4.6 mCrab). For S5 0716+714, S5 0836+710, and Mkn 6 the $3 \sigma$ upper limit is $\sim 10^{-3} \mathrm{ph} \mathrm{s}^{-1} \mathrm{~cm}^{-2}$ (6 mCrab). The extrapolation of the ISGRI spectra of S5 0836+710, Mkn 3 and Mkn 6 (see Sects. 2.1 and 2.2) to lower energies falls by a factor from 2 to 20 below the sensitivity of the JEM-X image. The JEM-X $3 \sigma$ upper limits for S5 0716+714, S5 0836+710, Mkn 3 and Mkn 6 are $6,10,8,6 \mathrm{mCrab}(5-20 \mathrm{keV})$, respectively.

No data were acquired with the Optical Monitor (Mas-Hesse et al. 2003).

\subsection{IBIS observation of S5 $0716+714$}

A first inspection of the IBIS/ISGRI data revealed the presence of high background, with some structures. However, in the final mosaic of all the available data (i.e., the weighted combination of the individual pointings), no systematic effects have been found.

S5 $0716+714$ is detected with signal-to-noise ratio $4.5 \sigma$ in the energy band $30-60 \mathrm{keV}$, for a count rate of $0.11 \pm$ 0.04 counts $\mathrm{s}^{-1}$. Since the source was better detected in the first part of the INTEGRAL observation, indicating that it was declining, we selected and accumulated the individual pointings of the early portion of the monitoring, for which the signal-tonoise ratio at the position of the blazar is larger than 1 . This reduces the useful exposure to a total of $84 \mathrm{ks}$, but allows us to improve the significance of the detection of S5 $0716+714$ to $6.5 \sigma$ in the $30-60 \mathrm{keV}$ energy range. No signal is detected above $60 \mathrm{keV}$.

Given the low signal-to-noise ratio, it was not possible to study the intra-orbit variability of the source, nor to extract a spectrum.

In order to evaluate the flux of the source we assumed a spectrum identical to that of the Crab nebula $(\Gamma=2.1)$ and scaled the normalization via the count rates ratio in the

\footnotetext{
${ }^{1}$ http://isdc. unige.ch/index.cgi?Soft+download
} 
$30-60 \mathrm{keV}$ range (Table 1). Note that our flux measurement is only representative of the higher state of the source during the observation, and not of the overall average state.

\subsection{Other AGNs in the IBIS field of view}

The large field of view of IBIS $\left(19^{\circ} \times 19^{\circ}\right.$ at half response $)$ allowed us to observe serendipitously other sources. Specifically, three additional AGNs are detected in the IBIS/ISGRI image with higher significance than our blazar target (Table 1), and up to $100 \mathrm{keV}$. Also for these sources, the detections in the individual pointings are not significant, and therefore it is not possible to study their intra-orbit variability. However, the signalto-noise ratio of the summed image is sufficiently high to allow the extraction of an average spectrum.

Since spectral reconstruction is very sensitive to the background correction in weak sources, we have extracted the spectra following two independent procedures, as recommended in the OSA guidelines ${ }^{2}$. For each source, we have obtained both a coadded spectrum from the spectra extracted from individual pointings, and a spectrum constructed from the imaging in the same energy bands used for spectral extraction. These are found to be consistent with each other in all sources. We have used the spectra obtained with the former method for spectral analysis. In order to apply the $\chi^{2}$ statistics, the spectral signal has been binned in intervals where the significance is at least $3 \sigma$.

In the energy range $\approx 60-80 \mathrm{keV}$ the IBIS spectra are affected by instrumental features (Terrier et al. 2003), that are not well modeled by the response matrix. The OSA V. 4 software reduces this problem only partially, therefore, considering also the relevance of the background relative to the flux levels of our sources, we conservatively excluded the above spectral region from the analysis.

We fitted single power-laws to the spectra of S5 0836+710 (Fig. 1) and Mkn 6 (Fig. 2) using the xspec package (v. 11.3.1). No systematic errors have been added in the fitting; we estimate the flux calibration uncertainties to be of the order of $\sim 10 \%$.

In Mkn 3, the spectral point at $\sim 100 \mathrm{keV}$ (see Fig. 3) makes a single power-law fit inadequate (the $\chi^{2}$ is 13 for 4 degrees of freedom); therefore we used a broken power-law, although the high energy index $\Gamma_{2}$ is obviously poorly constrained by the isolated point at $100 \mathrm{keV}$ (see contour plot in Fig. 4).

We have also tried a fit with a power-law plus an exponential cutoff. If the power-law photon index is left as a free parameter, it assumes an unreasonably flat value, $\Gamma \simeq-0.07$, inconsistent with the power-law index which best fits the spectral points at energies lower than $100 \mathrm{keV}, \Gamma=1.3$. A contour plot of the fitted photon index vs. high energy cut-off is shown in Fig. 5. By freezing instead the photon index to $\Gamma=1.3$, we obtain a cut-off energy of $\sim 87 \mathrm{keV}$ with an unacceptably high $\chi^{2}$ of 8.6 for 4 degrees of freedom. By adding in quadrature to the statistical uncertainties a systematic error as large as $25 \%$ we recover a $\chi^{2}=4$ for 4 degrees of freedom and the cut-off energy is $E=95_{-40}^{+105} \mathrm{keV}$.

\footnotetext{
${ }^{2}$ http://isdc.unige.ch/Soft/download/osa/osa_sw/ osa_sw-4.0/osa_issues-4.0.txt
}

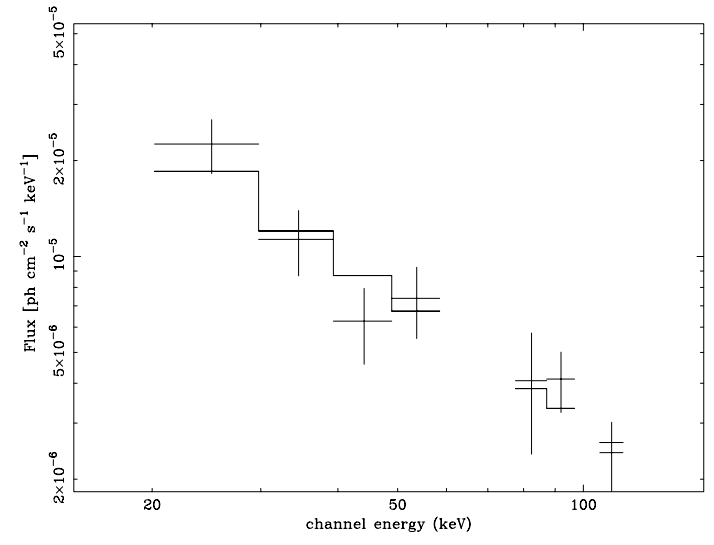

Fig. 1. IBIS/ISGRI spectrum of S5 0836+710. The overplotted steplike curve is the best fit single power-law.

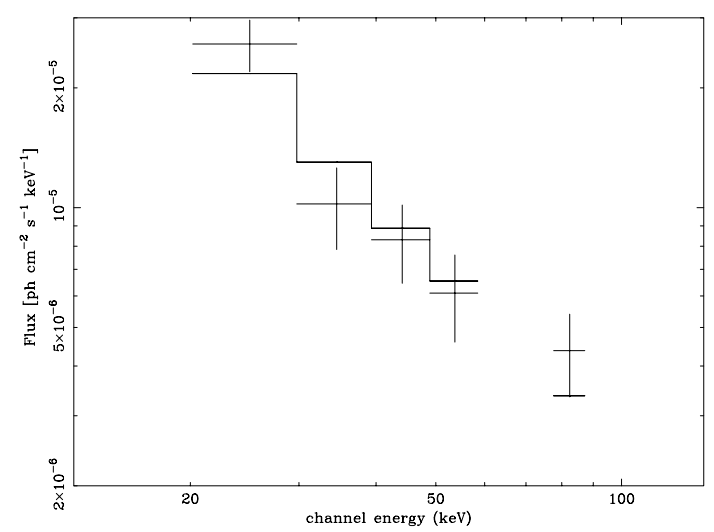

Fig. 2. IBIS/ISGRI spectrum of Mkn 6. The overplotted step-like curve is the best fit single power-law.

Therefore, we formally prefer the broken power-law rather than the cut-off power-law model. At best, the latter suggests a cut-off energy larger than $\sim 50 \mathrm{keV}$. We stress that the choice of the best interpretation of the spectral shape of Mkn 3 largely relies upon the point at $\sim 100 \mathrm{keV}$, that is very sensitive to background subtraction. A more robust spectral modeling must await a more accurate spectral measurement at these energies.

The results of the spectral analysis are reported in Table 1. The spectra, along with their best-fit models, are reported in Figs. 1-3.

\section{Discussion}

We observed the blazar S5 0716+714 with INTEGRAL while it was undergoing a major optical outburst and detected the source in a somewhat higher (about a factor of 2) gamma-ray state than observed in October 2000 (Tagliaferri et al. 2003). At that epoch the optical flux was slightly lower $(R \simeq 12.5)$ than that observed in March 2004 at maximum brightness. The BeppoSAX PDS spectrum suggests that the soft gamma-rays are due to inverse Compton scattering of relativistic particles (Tagliaferri et al. 2003; Giommi et al. 1999; see also the last column of Table 1, where the indices of the PDS spectra of our sources are reported for comparison with the present results). 
Table 1. Sources detected in the IBIS/ISGRI field of S5 0716+714.

\begin{tabular}{|c|c|c|c|c|c|c|c|c|c|c|c|}
\hline Object & AGN type & $z$ & $\mathrm{CR}^{a}$ & Flux $^{b}$ & Range $^{c}$ & $\Gamma_{1}^{d}$ & $\Gamma_{2}^{d}$ & $E_{b}^{e}$ & $\chi^{2}$ & d.o.f. & $\Gamma_{\mathrm{PDS}}^{f}$ \\
\hline S5 0716+714 & BL Lac & $\ldots$ & $0.36 \pm 0.07^{g}$ & 3.1 & $30-60$ & $\cdots$ & $\ldots$ & $\ldots$ & $\ldots$ & $\ldots$ & $1.6 \div 2.0$ \\
\hline S5 $0836+710$ & FSRQ & 2.172 & $0.54 \pm 0.05$ & 4.6 & $20-100$ & $1.3 \pm 0.3^{h}$ & $\ldots$ & $\ldots$ & 4.1 & 5 & $1.31 \pm 0.03$ \\
\hline Mkn 6 & Sy 1.5 & 0.019 & $0.49 \pm 0.06$ & 4.6 & $20-100$ & $1.5_{-0.4}^{+0.5}$ & $\ldots$ & $\ldots$ & 3.7 & 3 & $1.8 \pm 0.2$ \\
\hline Mkn 3 & Sy 2 & 0.013 & $0.82 \pm 0.05$ & 7.4 & $20-100$ & $1.3^{i .4}$ & $>2.5$ & $80 \pm 20^{h}$ & 2.5 & 3 & $1.8 \pm 0.1$ \\
\hline
\end{tabular}

${ }^{a}$ IBIS/ISGRI count rate in the detection energy range (Col. 6), in counts $\mathrm{s}^{-1}$.

${ }^{b}$ Fitted flux in the detection energy range (Col. 6), in $10^{-11} \mathrm{erg} \mathrm{s}^{-1} \mathrm{~cm}^{-2}$. Calibration uncertainties are $\sim 10 \%$.

${ }^{c}$ Energy interval to which count rates (Col. 4) and fluxes (Col. 5) are referred, in keV.

${ }^{d}$ Photon index: $f_{E} \propto E^{-\Gamma}$.

${ }^{e}$ Break energy, in keV.

${ }^{f}$ Photon index measured with the BeppoSAX PDS at previous epochs.

${ }^{g}$ Uncertainties on the count rates are $1 \sigma$.

${ }^{h}$ Uncertainties on the photon indices and break energy are $1.6 \sigma$.

${ }^{i} \Gamma_{1}$ has been frozen to the index of the single power-law which best fits the spectrum below $100 \mathrm{keV}, \Gamma=1.3 \pm 0.4$.

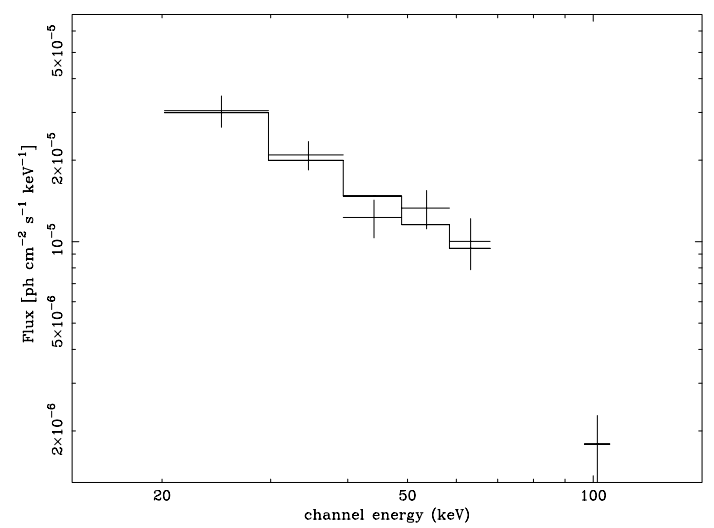

Fig. 3. IBIS/ISGRI spectrum of Mkn 3. The overplotted step-like curve is the single power-law which best fits the spectrum below $100 \mathrm{keV}$. The point at $100 \mathrm{keV}$ is clearly below this curve.

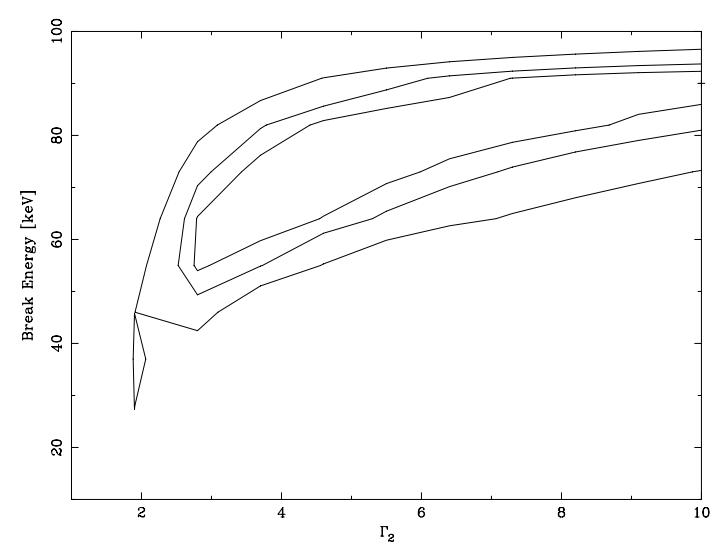

Fig. 4. Contour plot of break energy vs. photon index for the broken power-law model fit of Mkn 3 spectrum.

In the same field of our primary target we also detected the high redshift blazar S5 0836+710, belonging to the FSRQ subclass (Urry \& Padovani 1995), and two bright Seyfert galaxies, Mkn 3 and Mkn 6, all with brighter fluxes than S5 0716+714 (Table 1).

The spectral index of S5 $0836+710$ is consistent with that determined through CGRO BATSE (Malizia et al. 2000)

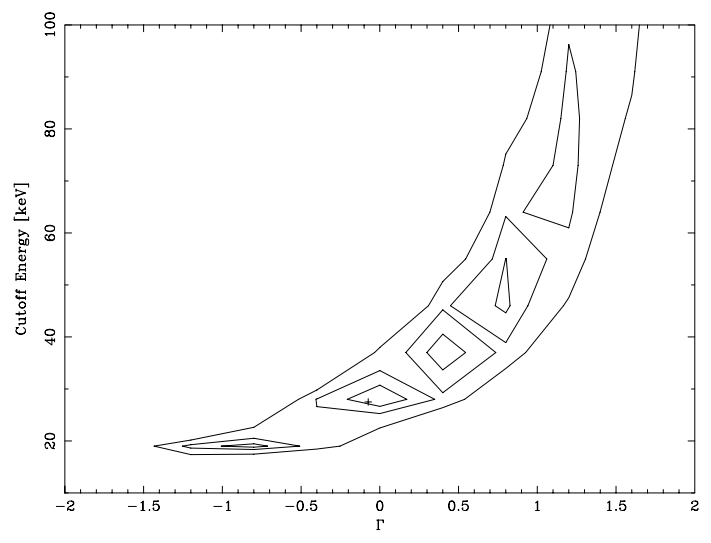

Fig. 5. Contour plot of cut-off energy vs. photon index for the cut-off power-law model fit of Mkn 3 spectrum.

and BeppoSAX PDS (Tavecchio et al. 2002) observations during higher emission states (our measured flux is a factor of $\sim 3$ lower than found by both BATSE and BeppoSAX). The flat spectral slope favours the interpretation of the high energy spectrum as Compton scattering of relativistic electrons off external radiation, as opposed to synchrotron-self Compton, in FSRQ (Sikora et al. 1994).

The IBIS/ISGRI spectral slope of the Seyfert Mkn 6 is flatter, although consistent with that measured by the BeppoSAX PDS (Malizia et al. 2003a; Immler et al. 2003), within our rather large errors. Similarly, the flux level in the IBIS/ISGRI observation is consistent with that measured by the PDS.

Our IBIS/ISGRI spectrum of Mkn 3 has an index consistent with that determined by $\operatorname{OSSE}(\Gamma \simeq 1.3$, Zdziarski et al. 2000) and suggests the presence of a high energy cut-off, at an energy approximately compatible with those of the breaks detected in other Seyfert objects and radiogalaxies by OSSE and BeppoSAX, although on the lower energy side with respect to the average break energy determined by BeppoSAX for Seyfert 2 galaxies (Johnson et al. 1997; Grandi et al. 1998; Piro et al. 1998; Nicastro et al. 2000; Zdziarski \& Grandi 2001; Zdziarski et al. 2000; Gondoin 2001; De Rosa et al. 2002; Malizia et al. 2003b; Deluit \& Courvoisier 2003). However, no 
evidence of a cut-off was found in Mkn 3 by Cappi et al. (1999) at least up to $150 \mathrm{keV}$, the maximum energy at which the source was detected by the BeppoSAX PDS. Therefore, while considering our detection of a cut-off tentative, we cannot exclude a variable cut-off energy. We note in fact that the flux detected by the PDS was $\sim 30 \%$ higher than that detected by IBIS, and the spectral slope $(\Gamma \simeq 1.8)$ marginally steeper (see Cappi et al. 1999). A similar correlation of cut-off energy and spectral steepness has been observed with BeppoSAX in the Seyfert 1 NGC 5548 (Nicastro et al. 2000; Petrucci et al. 2000). A better exposed INTEGRAL ISGRI and/or SPI spectrum would be necessary to confirm our finding.

INTEGRAL has so far significantly detected a number of radio-quiet and radio-loud AGNs (Bassani et al. 2004; Beckmann et al. 2004; Bird et al. 2004), among which the brightest known radio-loud AGN, 3C 273 (Courvoisier et al. 2003). Our observation of S5 0716+714 and serendipitous detection of three additional AGNs proves INTEGRAL to be effective, even with relatively short exposure observations, in the study of bright extragalactic sources at high Galactic latitudes and underscores the importance of instruments with a large field of view and good angular resolution for the investigation of gamma-ray-loud AGNs. S5 $0836+710$ is the second highest redshift AGN detected by INTEGRAL, after PKS 1830-21 at $z=2.507$ (Bassani et al. 2004), which indicates that INTEGRAL can also play a role in exploring the high redshift universe.

Acknowledgements. We thank S. J. Wagner for providing us with preliminary information on the RXTE monitoring of S5 0716+714, which helped in scheduling our INTEGRAL observation, and the staff at the INTEGRAL Science Operation Center and Science Data Center for their assistance in data acquisition and analysis, and in particular A. Gros, P. Kretschmar, A. Parmar, K. Pottschmidt, R. Williams. The anonymous referee is acknowledged for a constructive report. We acknowledge the EU funding under contract HPRCN-CT-200200321 (ENIGMA network). EP and LF acknowledge the Italian Space Agency (ASI) for financial support.

\section{References}

Bassani, L., Malizia, A., Stephen, J. B., et al. 2004, Proceedings of the V INTEGRAL Workshop, Munich 16-20 February 2004, ESA SP-552, in press [arXiv: astro-ph/0404442]

Beckmann, V., Gehrels, N., Favre, P., et al. 2004, ApJ, in press [arXiv: astro-ph/0406553]
Bird, A. J., Barlow, E. J., Bassani, L., et al. 2004, ApJ, 607, L33

Cappi, M., Bassani, L., Comastri, A., et al. 1999, A\&A, 344, 857

Courvoisier, T. J.-L., Walter, R., Beckmann, V., et al. 2003a, A\&A, 411, L53

Courvoisier, T. J.-L., Beckmann, V., Bourban, G., et al. 2003b, A\&A, 411, L343

Deluit, S., \& Courvoisier, T. J.-L. 2003, A\&A, 399, 77

De Rosa, A., Piro, L., Fiore, F., et al. 2002, A\&A, 387, 838

Di Cocco, G., Caroli, E., Celesti, E., et al. 2003, A\&A, 411, L189

Diehl, R., Baby, N., Beckmann, V., et al. 2003, A\&A, 411, L117

Giommi, P., Massaro, E., Chiappetti, L., et al. 1999, A\&A, 351, 59

Goldwurm, A., David, P., Foschini, L., et al. 2003, A\&A, 411, L223

Gondoin, P., Barr, P., Lumb, D., et al. 2001, A\&A, 378, 806

Grandi, P., Haardt, F., Ghisellini, G., et al. 1998, ApJ, 498, 220

Haardt, F., Maraschi, L., \& Ghisellini, G. 1997, ApJ, 476, 620

Immler, S., Brandt, W. N., Vignali, C., et al. 2003, AJ, 126, 153

Johnson, W. N., McNaron-Brown, K., Kurfess, J. D., et al. 1997, ApJ, 482, 173

Lebrun, F., Leray, J. P., Lavocat, P., et al. 2003, A\&A, 411, L141

Lund, N., Budtz-Jørgensen, G., Westergaard, N. J., et al. 2003, A\&A, 411, L231

Malizia, A., Bassani, L., Dean, A. J., et al. 2000, ApJ, 531, 642

Malizia, A., Bassani, L., Capalbi, M., et al. 2003a, A\&A, 406, 105

Malizia, A., Bassani, L., Stephen, J. B., et al. 2003b, ApJ, 589, L17

Mas-Hesse, J. M., Giménez, A., Culhane, L., et al. 2003, A\&A, 411, L261

Nicastro, F., Piro, L., De Rosa, A., et al. 2000, ApJ, 536, 718

Petrucci, P. O., Haardt, F., Maraschi, L., et al. 2000, ApJ, 540, 131

Piro, L., Nicastro, F., Feroci, M., et al. 1998, Nucl. Phys. B (Proc. Suppl.), 69/1-3, 481

Sikora, M., Begelman, M. C., \& Rees, M. J. 1994, ApJ, 421, 153

Svensson, R. 1996, ApJS, 92, 585

Tagliaferri, G., Ravasio, M., Ghisellini, G., et al. 2003, A\&A, 400, 477

Tavecchio, F., Maraschi, L., Ghisellini, G., et al. 2000, ApJ, 543, 535

Terrier, R., Lebrun, F., Bazzano, A., et al. 2003, A\&A, 411, L167

Ubertini, P., Lebrun, F., Di Cocco, G., et al. 2003, A\&A, 411, L131

Ulrich, M.-H., Maraschi, L., Urry, C. M. 1997, ARA\&A, 35, 445

Urry, C. M., \& Padovani, P. 1995, PASP, 107, 803

Vedrenne, G., Roques, J.-P., Schönfelder, V., et al. 2003, A\&A, 411, L63

Villata, M., Raiteri, C. M., Kurtanidze, O. M., et al. 2004, A\&A, 421, 103

Westergaard, N. J., Kretschmar, P., Oxborrow, C. A., et al. 2003, A\&A, 411, L257

Winkler, C., Courvoisier, T. J.-L., Di Cocco, G., et al. 2003, A\&A, 411, L1

Zdziarski, A. A., \& Grandi, P. 2001, ApJ, 551, 186

Zdziarski, A. A., Poutanen, J., \& Johnson, N. W. 2000, ApJ, 542, 703 